\title{
Les représentations du livre numérique chez les professionnels de l'information-documentation
}

Information Professionals' Representations of Electronic Books: The Case of Public and School Librarians

Fabrice Pirolli et Eric Heilmann

\section{OpenEdition}

\section{Journals}

Édition électronique

URL : https://journals.openedition.org/edc/6011

DOI : 10.4000/edc.6011

ISSN : 2101-0366

Éditeur

Université de Lille

Édition imprimée

Date de publication : 1 décembre 2014

Pagination : $75-90$

ISBN : 978-2-917562-12-3

ISSN : $1270-6841$

\section{Référence électronique}

Fabrice Pirolli et Eric Heilmann, « Les représentations du livre numérique chez les professionnels de

l'information-documentation ", Études de communication [En ligne], 43 | 2014, mis en ligne le 01 décembre 2014, consulté le 21 septembre 2021. URL : http://journals.openedition.org/edc/6011 ; DOI : https://doi.org/10.4000/edc.6011

(c) Tous droits réservés 


\section{FABRICE PIROLLI ET ERIC HEILMANN LES REPRÉSENTATIONS DU LIVRE NUMÉRIQUE CHEZ LES PROFESSIONNELS DE L'INFORMATION-DOCUMENTATION}

Peu à peu le livre numérique trouve sa place dans l'offre culturelle française. Même si le développement de ce marché semble plus lent que dans d'autres pays - comme le montre encore dernièrement le rapport Wischenbart (2013) - il est aujourd'hui une réalité qui modifie les pratiques, les attentes et la position des différents acteurs impliqués dans les métiers du livre (Robin, 2011). Parmi les sphères professionnelles directement concernées, celle de l'informationdocumentation occupe une place particulière. En effet, bibliothécaires et documentalistes sont directement concernés par une évolution qui, si elle n'en est qu'à son commencement, peut à terme remettre en question leurs missions et la nature de leur profession. Les mutations entrainées par l'arrivée massive du numérique dans l'univers du livre ne semblent pas de prime abord remettre en cause la position de médiateur qu'occupent libraires, bibliothécaires et documentalistes. Mais, selon Marcel Gauchet (2009), la réalité serait tout autre. «De manière sourde, souligne-t-il, ces professions de la chaîne du livre sont peut-être les plus profondément concernées par cette crise de la médiation. Parce que la technique met à l'ordre du jour l'horizon utopique de leur disparition ». 
La question de l'offre de livres numériques en bibliothèques n'est pas nouvelle. De nombreuses initiatives témoignent de son importance et de son actualité. On peut citer à titre indicatif les travaux menés par

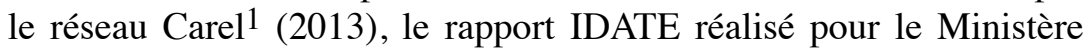
de la culture et de la communication (2013) ainsi que les nombreux événements professionnels (tables-rondes, symposiums, séminaires...) organisés régulièrement autour de cette thématique. Cela étant, si les pratiques de lecture (Opinionway, 2013), les pratiques d'achats (Boullier et Crépel, 2013), les modèles éditoriaux (Robin, 2011), et diverses expériences de prêt de livres numériques en bibliothèques (MOTif, 2011) ont fait l'objet de travaux spécifiques, peu d'études portent sur la manière dont les professionnels de l'informationdocumentation perçoivent ces évolutions. Que ces praticiens soient utilisateurs ou non de dispositifs de lecture numérique, qu'ils aient été associés ou non dans leur milieu professionnel à une réflexion ou à la mise en place d'actions de médiation portant sur le livre numérique, comment se représentent-ils cette réalité au-delà des discours institutionnels, prospectifs et commerciaux ? De quelles manières appréhendent-ils aujourd'hui ces contenus et ces dispositifs nouveaux ? Comment et pourquoi les ont-ils déjà intégrés ou non à leurs pratiques professionnelles ? Dans quelles mesures considèrentils que leur propre métier sera modifié par cette évolution ? L'arrivée du livre numérique est-elle ressentie comme une menace ou comme une opportunité ? En quoi, selon eux, les rapports aux publics pourraient en être modifiés?

Cette étude vise précisément à cerner les représentations du livre numérique et les évolutions qu'il pourrait induire dans les pratiques professionnelles auprès de deux catégories spécifiques d'acteurs : des bibliothécaires en poste dans des bibliothèques de lecture publique et des enseignants-documentalistes exerçant en Centres de Documentation et d'Information (CDI) au sein d'établissements scolaires, collèges ou lycées. Ces deux catégories de professionnels évoluent dans des environnements présentant des spécificités propres, tant du point de vue réglementaire que du point de vue des objectifs, des publics et des moyens. Cependant, tous ont en commun d'accomplir au quotidien un ensemble de tâches et de missions s'inscrivant dans le champ disciplinaire de l'information-

1 Association en faveur de la Coopération pour l'Accès aux Ressources Electroniques en Bibliothèques. 
documentation. Après avoir brièvement présenté le cadre de cette étude et l'approche méthodologique retenue, nous tenterons de mettre en lumière les représentations associés au livre numérique chez les professionnels interrogés, puis analyserons son impact - réel ou supposé - sur les pratiques professionnelles.

\section{Contexte et méthodologie de la recherche}

Le travail présenté ici s'inscrit dans le cadre plus général d'un projet de recherche portant sur le livre numérique cofinancé par le Conseil Régional de Bourgogne et l'université de Bourgogne. A ce titre, le recueil d'informations a eu lieu auprès de professionnels exerçant dans l'Yonne, la Côte-d'Or, la Nièvre ou la Saône-et-Loire. Nous avons suivi une approche qualitative fondée sur la réalisation d'entretiens semi-directifs. La grille d'entretien a été conçue dans le cadre d'un séminaire de recherche auquel participaient des étudiants préparant le capes documentation (master $2^{\mathrm{e}}$ année). Au fil de son élaboration, quatre thématiques principales ont émergé : les éléments de représentation du livre électronique; les usages personnels et professionnels de livres numériques; la perception des demandes et des attentes des publics; les perspectives d'évolution des pratiques professionnelles et des métiers. L'approche retenue pour le recueil d'information est donc uniquement de nature déclarative. D'autres types d'approches, fondées par exemple sur l'observation, pourraient à l'avenir compléter notre démarche mais elles n'ont pas, à ce jour, été mises en œuvre.

Les entretiens ont été menés en majorité par nos étudiants auprès de professionnels de la région entre octobre et décembre 2013. Après transcription et validation, vingt-sept entretiens ont été retenus (quatorze bibliothécaires et treize enseignants-documentalistes) pour constituer l'échantillon de l'enquête ${ }^{2}$. Nous avons veillé à ce que les professionnels interrogés soient géographiquement répartis sur des territoires de natures variées (urbains, périurbains et ruraux) et exercent dans des établissements de tailles différentes. Les personnes

2 L'objectif de ce séminaire était de familiariser les étudiants inscrits dans un cursus professionnel à une démarche de recherche. Le travail réalisé par chaque étudiant (conduite de trois entretiens, transcription puis brève analyse) a fait l'objet d'une évaluation au terme du $1^{\mathrm{er}}$ semestre de l'année universitaire. Les auteurs tiennent à les remercier ici pour leur contribution à cette étude. 
retenues pour ces entretiens n'ont pas été sélectionnées en raison d'une implication particulière dans le développement d'une offre numérique au sein de leur établissement, ni de la manifestation d'un intérêt personnel fort pour l'usage de dispositifs de lecture numérique. Les retranscriptions des différents entretiens ont fait ensuite l'objet d'une analyse de contenu présentée ici.

\section{Le livre numérique, définition et pratiques personnelles}

La majorité des professionnels interrogés définit le livre numérique par sa forme: "c'est la version numérique d'un écrit papier» (V. M. professeur documentaliste en lycée) ; «c'est un livre dont le contenu a été numérisé» (C. L. professeur documentaliste en lycée) ; "il est semblable à un livre papier sauf qu'il est édité et diffusé en version numérique » (M. C. assistante de conservation en médiathèque) ou encore, comme le souligne sobrement C.C. (professeur documentaliste en collège), «je n'ai pas de définition plus précise que celle-là, c'est une histoire de format». C'est donc la forme prise par le texte, plus que l'objet matériel lui-même, qui constitue l'élément central de ces définitions. Elle conditionne le mode de lecture car celle-ci est impossible sans un dispositif doté d'un écran. Et tous citent spontanément les différents supports disponibles sur le marché : liseuses, tablettes, ordinateurs et téléphones portables. Ainsi, le livre numérique entendu comme un format transcende toutes ses possibles matérialisations.

Les personnes interrogées ont-elles une expérience de la lecture numérique ? Les réponses sont très tranchées. Un tiers des personnes interrogées répondent par la négative. Ainsi, J. L. bibliothécaire dans une zone rurale, affirme: "je n'en éprouve pas le besoin, la lecture sur écran ça ne m'intéresse pas». Cette absence d'intérêt prend parfois la forme d'une véritable répulsion pour l'objet: «je n'ai pas envie de lire sur un écran, j'ai déjà assez mal à la tête comme $c ̧ a »$ (A. G. professeur documentaliste en collège). Pour expliquer le non-usage d'une liseuse, certains évoquent une relation plus intime avec les livres imprimés. Professeur documentaliste en collège, A.C. parle d'un «rapport plus personnel» avec le livre papier; "le livre numérique ne transmet pas la même sensation au toucher, remarque N. J. professeur documentaliste en lycée, il ne possède pas cette odeur particulière du livre papier ». Une autre collègue 
décrit sa difficulté «à entrer dans le texte » avec une liseuse. Deux bibliothécaires, amateurs de bande dessinée, évoquent quelques expériences en la matière mais disent finalement y avoir renoncé par «manque de lisibilité ». Fait remarquable ici, la discordance existante entre la définition donnée au livre numérique (un format) et les modalités de sa lecture qui mobilise nécessairement un matériel spécifique (un objet doté d'un écran) : l'objet-support ressurgit dans le discours des non-usagers lorsqu'ils expliquent leur refus ou leur réticence à l'utiliser.

Du côté des usagers, l'objet utilisé par le plus grand nombre est la liseuse. Elle est présentée comme un objet «pratique », «léger » et «peu encombrant», propice à un emploi nomade. Son principal atout? Plusieurs d'entre eux soulignent sa capacité de stockage: « on peut avoir toute sa bibliothèque sur soi » (V. D. bibliothécaire) ; «c'est bien plus pratique que de se trimballer avec sa valise de bouquins » (C. D. directrice adjointe d'une médiathèque en zone urbaine). D'autres pointent des fonctionnalités particulières de la liseuse pour expliquer leur intérêt : «je lis dans le train ; j'ai un seul objet dans la main, c'est léger; on peut régler la taille des caractères, on n'a pas besoin de mettre ses lunettes » (M. S. directrice d'une bibliothèque en zone urbaine) ; "je voulais que ce soit rétro éclairé pour pouvoir lire au lit sans allumer la lumière, j'ai donc choisi ce modèle là, je l'utilise tout le temps maintenant» (A. P. professeur documentaliste dans un lycée).

Capacité de stockage et confort de lecture font de la liseuse le support privilégié par nos interlocuteurs dans un cadre personnel où la tablette et plus encore l'ordinateur n'ont pas leur place pour une lecture associée au plaisir ou à la détente. Tablette et ordinateur sont toujours associés au web et à la recherche documentaire, c'est-à-dire à un cadre professionnel. Le support technique de la lecture est donc systématiquement associé à un environnement précis. Il délimite une frontière nette entre cadre personnel et cadre professionnel. A cette première frontière s'en ajoute une autre, parfaitement claire, qui délimite les contenus appropriés ou non à la lecture sur liseuse. D'un côté sont cités les romans et les œuvres classiques (entrés dans le domaine public), de l'autre figurent notamment les bandes dessinées et les beaux livres dont la taille et la présence d'illustrations sont considérées comme impropres à une consultation sur liseuse.

En s'appuyant sur leurs pratiques personnelles, nos interlocuteurs émettent des critiques à l'égard de l'usage de la liseuse, mais 
elles sont rares et toujours formulées par des bibliothécaires. La première concerne le format des livres numériques, perçu comme un frein susceptible de bloquer l'accès à tel ou tel contenu. «Avec un Kindle, on ne peut acheter que sur Amazon; avec un Kobo, nous sommes prisonniers du catalogue de la Fnac» (M. C.). La seconde concerne l'absence de don possible du livre qui a plu. "C'est un peu contradictoire, souligne M. S.,j'aime acheter mes propres livres et les donner à d'autres personnes après. Il m'arrive de racheter plusieurs fois le même livre pour le donner à des personnes différentes, pour le faire partager. Avec un e-book, on ne peut pas en faire profiter les gens de la même façon ». Curieusement, comme on le verra plus loin, cette dernière réserve n'est pas reprise par nos interlocuteurs lorsqu'ils sont interrogés sur l'évolution de leurs pratiques professionnelles, notamment à l'égard du prêt d'ouvrages en bibliothèque ou CDI. Quel doit être son objet? S'agit-il du livre numérisé et/ou de la liseuse ? Selon quelles modalités ? Des questions qui demeurent sans réponse ici.

\section{Les pratiques professionnelles à l'épreuve du livre numérique}

Les ressources numériques et la lecture sur écran ont fait leur entrée en bibliothèques et centres de documentation depuis maintenant de nombreuses années (Bertrand, 2004). Les pratiques liées au numérique ont également contribué à modifier profondément les habitudes des praticiens de la documentation (Pirolli, 2010). Cependant, le développement du livre numérique comme alternative aux supports écrits pour certaines catégories de documents - documentaires, romans, œuvres classiques - restées jusqu'alors à l'écart de cette évolution amorce de nouveaux changements dans les pratiques professionnelles du domaine de l'information-documentation. Afin de cerner les contours de ces nouvelles pratiques, nous nous sommes intéressés, d'une part, à la place du livre numérique dans les organismes étudiés, d'autre part, au point de vue des professionnels sur les évolutions futures de leur métier et leur vision de l'avenir des centres de ressources documentaires et des bibliothèques. 


\section{La place du livre numérique dans les organismes étudiés}

Le taux d'équipement en matière de dispositifs mobiles de lecture, tablettes ou liseuses, des structures étudiées est aujourd'hui très faible. Sur l'ensemble des organismes consultés, seuls quatre ont effectivement fait l'acquisition de matériels spécifiques, avec une moyenne de cinq dispositifs par établissement. Dans ce cas, l'acquisition du matériel est perçue comme une première étape à visée exploratoire et non véritablement comme le commencement d'une réorganisation de l'offre de lecture ou des politiques d'acquisition. La question du matériel semble avoir été abordée indépendamment de la question des contenus : "on s'est lancés et on a acheté, mais on ne sait pas quel genre d'offre proposer» (V. D. bibliothécaire); «on s'est dit que, même si on ne proposait pas de livres numériques, ça serait bien au moins que les usagers puissent se familiariser avec les supports »(C. D. bibliothécaire). Aucune des organisations concernées n'a procédé à l'acquisition d'ouvrages sous forme numérique, seuls des contenus libres de droits ont été téléchargés. En l'état, dans le cadre professionnel, la composante technique du dispositif prend donc le pas sur l'offre documentaire ou littéraire. La question du livre numérique y est systématiquement considérée, au moins dans un premier temps, du point de vue des dispositifs techniques.

Les personnes ayant joué un rôle actif dans la mise en place d'une offre de lecture numérique expliquent que leur démarche relève davantage de la curiosité que d'une volonté prescriptive ou d'une conviction personnelle forte. Il est intéressant de noter que les professionnels les plus actifs sur ce plan n'ont pas suivi de formations spécifiques et qu'ils agissent de manière empirique. A l'inverse, les personnes ayant suivi des formations consacrées au livre numérique n'ont pas encore mis en place d'offre spécifique et n'expriment pas, pour la plupart, la volonté d'en développer à court terme. Dans les établissements qui n'ont pas encore entrepris de démarches de cette nature, les raisons les plus couramment mises en avant sont liées au budget et à la maintenance des dispositifs. Cependant, elles n'expliquent pas à elles seules cette situation. Sur ce point, on constate une forte disparité entre les bibliothèques et les CDI.

Pour les bibliothèques publiques, le manque d'intérêt supposé des usagers pour la lecture numérique est mentionné comme étant la principale raison. Le peu de demandes émanant des usagers, ainsi 
que la composition sociodémographique des publics fréquentant les établissements, amènent les professionnels à considérer l'offre en lecture numérique comme peu pertinente en regard de leurs missions. L'âge moyen des abonnés est fréquemment présenté comme un élément justifiant à lui seul le peu d'importance que pourrait revêtir le développement d'une offre en matière de livre numérique. «La population de la ville est vieillissante, explique ainsi F. H. bibliothécaire, les gens qui viennent à la bibliothèque ici ont quand même, pour la grande majorité, entre 60 et 85 ans et ce sont des gens qui utilisent très peu l'informatique ». "S'il n'y a pas d'offre, affirme M.C. assistante de conservation, ce n'est pas par opposition, c'est parce qu'il faut voir ce qui est possible au niveau du budget et puis quel est l'intérêt des gens aussi, il n'est vraiment pas sûr que ce soit une nécessité dans l'immédiat ».

L'idée de développer une offre nouvelle intégrant une composante numérique afin d'attirer de nouveaux publics n'est jamais directement mise en avant. Le livre numérique n'apparaît donc pas, aux yeux des professionnels interrogés, comme une priorité à moyen terme dans la mesure où son développement s'accomplirait au détriment du fonds papier. Il s'inscrit davantage dans une continuité de développements, initiées par l'arrivée des écrans et de l'internet (Bertrand, 2004), qui se poursuivront au rythme de la diffusion des technologies dans le grand public. Ainsi, pour A. J. bibliothécaire, c'est bien le numérique dans son ensemble qui questionne les professionnels : " $L a$ dématérialisation des supports ne s'arrête pas seulement au livre numérique. Elle concerne aussi l'animation... Mais pas seulement. La numérisation doit s'étendre à l'ensemble des ressources et permettre de développer l'aspect participatif des ressources en ligne ».

La posture la plus couramment constatée est donc celle d'un observateur attentif et curieux qui ne se sent pas, dans l'immédiat, directement concerné sur le plan professionnel. Dans le domaine de la lecture de loisir, il apparaît ainsi plus légitime de suivre les évolutions en cours plutôt que de tenter d'initier ou d'accompagner les changements. Ainsi, F. H. bibliothécaire et utilisatrice d'une liseuse à titre personnel, reconnait partager ses propres livres numériques avec quelques abonnés équipés, en dehors de tout cadre institutionnel et réglementaire. Toutefois, cette professionnelle est convaincue du peu d'intérêt que présenterait une offre de lecture publique numérique pour sa bibliothèque. 
Concernant les enseignants-documentalistes, la question de l'intérêt pédagogique est centrale dans les réflexions menées autour de l'offre numérique. Sont particulièrement mis en avant les rapports parfois difficiles des élèves à la lecture, notamment dans le cadre normé et prescriptif de l'institution scolaire. Deux perceptions opposées peuvent être identifiées sur ce point. Pour une première catégorie de professionnels, le livre numérique est une opportunité à saisir pour susciter un nouvel intérêt de la part élèves : "ça peut attirer de nouveaux lecteurs» (A. C. professeur documentaliste en collège) ; "pour attirer ceux qui ne lisent pas du tout, c'est peutêtre un moyen» (A. P. professeur documentaliste en lycée). Le changement de support est présenté comme potentiellement initiateur à lui seul d'un changement des comportements. En revanche, de nombreux enseignants sont convaincus que l'utilisation dans le cadre scolaire de dispositifs de lecture numérique n'a pas un impact significatif sur l'attitude des élèves: "je ne crois pas que cela changera grand-chose à la motivation, ou au manque de motivation, de nos élèves concernant la lecture » (N. J. professeur documentaliste en lycée). Certains tiennent d'ailleurs un discours très critique à l'encontre de cette approche. En la matière, la méfiance par rapport à des choix qui pourraient être imposés, quelles qu'en soient les raisons, est également très présente.

L'expression de craintes liées aux effets de modes et aux discours institutionnels est donc bien réelle. «L'Éducation nationale s'intéresse aux tablettes, souligne C. R. professeur documentaliste en collège, parce que c'est le dernier gadget à la mode... en attendant le suivant». L'idée d'un primat de la technologie sur les autres composantes du métier est également perçue comme l'une des conséquences négatives de cette évolution «Ma peur, ajoute-t-il, c'est que l'on soit réduit au côté technique du numérique, qui, pour moi, est le côté le moins important».

La prise de distance par rapport à la dimension technique des dispositifs est assez nette, d'autant que les élèves sont de plus en plus utilisateurs à titre personnel de tablettes numériques. Or, si leur connaissance de l'outil est bien réelle, les utilisations en dehors du cadre des loisirs restent limitées ou sont inexistantes. Le rôle de l'enseignant-documentaliste doit être alors davantage porté sur la sélection des sources, l'organisation des contenus et la scénarisation des usages dans un contexte pédagogique. En cela, l'intégration progressive d'une offre de lecture numérique en CDI s'inscrirait 
naturellement dans la mission d'éducation à la culture informationnelle qui est déjà assignée aux enseignants-documentalistes (Serres, 2008). $\mathrm{Si}$, dans le cas des bibliothèques, la démarche d'acquisition de matériels est souvent considérée comme le point d'entrée dans l'univers du livre numérique, cette position n'est pas aussi nette au sein des CDI étudiés. L'achat de dispositifs de lecture est perçu majoritairement de façon négative : sont ainsi évoqués les coûts, l'obsolescence rapide des matériels et les problèmes de maintenance. D'une façon générale, l'attente de retours d'expériences de terrain émanant d'autres établissements est très forte : «Ce qui m'aiderait à me lancer, déclare A. G. professeur documentaliste en collège, c'est que quelqu'un me dise : voilà, ça, ça marche bien, c'est moins cher et aussi efficace ».

\section{Impacts supposés sur les pratiques professionnelles}

Parmi les praticiens interrogés, peu de personnes manifestent la volonté de jouer dans leur milieu professionnel un rôle actif pour le développement de l'offre de livres numériques. Cela étant, celle-ci leur apparaît, à de très rares exceptions près, inévitable sur le long terme. Le livre numérique n'est jamais perçu comme devant se substituer au livre papier mais comme un complément de ce dernier qui pourrait enrichir les catalogues et l'offre documentaire. Lecture numérique et lecture papier seront appelées à coexister en fonction des contextes d'utilisations, des besoins et des lecteurs, comme l'exprime M. S. bibliothécaire: "Je ne crois pas que le numérique puisse remplacer le papier, les deux choses se côtoieront. Si on prend l'exemple de la lettre que l'on envoie par la poste et de celle que l'on envoie par mail, l'une ira plus vite mais ce n'est pas la même chose. Si on vous disait d'un jour à l'autre comme ça, on va supprimer le courrier postal, ça ne serait pas possible ».

Le livre numérique n'est pas perçu comme l'élément déclencheur d'une profonde évolution des métiers et des pratiques professionnelles en bibliothèque. "Le métier ne va pas forcément changer mais il est nécessaire de s'adapter, de maîtriser le sujet pour être capable de pouvoir renseigner le public, être au courant des pratiques» (M.C. assistante de conservation). Les professionnels s'accordent à penser que les principaux rôles qu'ils peuvent tenir dans le cadre des politiques d'acquisition, des actions de médiation et des 
animations à destination des publics seront simplement modifiés dans leurs modalités d'exécution. "Cela ne change pas les objectifs, cela ne change pas la finalité du métier, affirme C. J. professeur documentaliste en collège. Ça changera surtout certaines pratiques au quotidien ».

Les bibliothécaires interrogés n'expriment pas explicitement d'inquiétudes par rapport à l'avenir de leur profession en raison de l'arrivée du livre numérique. Cette position peut paraitre surprenante et même opposée aux constats dressés par d'autres travaux ayant traité de cette question (Collet et Pelissier, 2012). Elle est fondée sur une double conviction largement partagée : celle de la pérennité du support papier dont la disparition reste illusoire et celle de l'importance du rôle social tenu par les bibliothèques de lecture publique. La bibliothèque a vocation à demeurer un lieu de rencontres, d'échanges et de culture. Le modèle des bibliothèques «troisième lieu » (Oldenburg, 2001 ; Black, 2008) est, à ce titre, très fréquemment mis en avant.

La vision des enseignants-documentalistes est plus mitigée. Le développement éventuel d'une offre de livres numériques à destination des élèves ne fait pas l'objet d'inquiétudes particulières dans la mesure où de nombreux enseignants considèrent avoir déjà largement intégré les ressources numériques existantes (sites web, presse en ligne, etc.) dans l'exercice quotidien de leur métier. Le livre numérique en est une nouvelle déclinaison. Cependant, l'évolution, initiée par la circulaire de rentrée de 2012 qui vise à transformer progressivement les CDI en Centres de Connaissances et de Culture ou « $3 \mathrm{C} »^{3}$, suscite de nombreuses interrogations sur l'évolution des métiers. Présentés comme étant des projets éducatifs et pédagogiques à part entière, il est officiellement précisé que «les $3 C$ font cohabiter le livre et le numérique ». Pour la majorité de nos interlocuteurs, cette perspective est perçue comme une évolution naturelle et plutôt positive, même si elle demeure à ce jour encore floue. Elle génère néanmoins quelques craintes, notamment sur le rôle que devront tenir les enseignants et la façon d'appréhender et d'accompagner ce mouvement. "On nous dit que l'un des points principaux c'est justement la culture numérique, on nous rabâche beaucoup 'culture du numérique', 'culture du numérique', mais je trouve que derrière on ne met que de la technicité et de la technologie [...]. Moi, ce

3 Circulaire de rentrée $n^{\circ} 2012-056$ du 27/03/2012 « Orientations et instructions pour la préparation de la rentrée $2012 »$ annexe 7. 
que je vois derrière, c'est la pédagogie de tout ce qui touche le numérique, or on ne parle plus de pédagogie, on parle de numérique » (C. R. professeur documentaliste en collège).

L'introduction progressive de tablettes numériques dans le cadre d'enseignements scolaires autres que ceux directement liés à la documentation est parfois présentée comme problématique. Elle relève trop rarement d'actions coordonnées transversales au niveau des établissements. Or, plusieurs personnes interrogées mettent en avant qu'une approche plus globale impliquant les enseignantsdocumentalistes permettrait de contextualiser et de problématiser les usages prescrits. Citons par exemple le cas de A. C. documentaliste en collège, qui constate que l'introduction de tablettes dans son établissement n'a pas donné lieu à la prise en compte d'enjeux liés à l'information-documentation. En l'espèce, l'apport du numérique serait même négatif dans la mesure où les élèves concernés fréquentent moins le CDI : "Nous avons ici une classe 'cartable numérique' mais les documentalistes ne sont pas impliquées. Du coup, je ne vois pas les élèves de cette classe, ils ne viennent pas au CDI avec leurs professeurs puisqu'ils ont des ressources avec eux. Les élèves de cette classe fréquentent le CDI en autonomie mais je ne les vois pas avec leur tablette. Ils ne me font pas de demandes particulières pour des livres numériques ».

Sont ainsi maintenues des frontières entre apprentissages disciplinaires, éducation à la culture informationnelle et formation au numérique, alors même que les technologies impliquées pourraient, au contraire, ouvrir d'intéressantes perspectives transdisciplinaires. Les principales inquiétudes exprimées ne concernent pas l'arrivée du livre numérique dans les CDI, ni la dimension technologique qu'elle suppose, mais bien davantage la pérennité du statut professionnel des praticiens concernés.

\section{Conclusion}

$\mathrm{Au}$ terme de cette étude, plusieurs constats s'imposent. Tout d'abord, la question du matériel nécessaire à la consultation des livres numériques, qu'elle soit perçue positivement ou négativement, prend le pas sur la question des contenus. Même lorsque l'importance de ces derniers est mise en avant dans les discours, dans les faits, parmi tous nos interlocuteurs, aucune réflexion sur l'offre (livres numériques 
consultables sur place, téléchargeables, formats à privilégier, intermédiaires, cadre légal, etc.) n'est menée en profondeur. Il était par exemple envisageable que les personnes interrogées, en s'appuyant sur leur expérience personnelle, appréhendent la liseuse non pas comme un simple support de lecture mais comme une bibliothèque («une valise de bouquins» dit l'une d'elle) dont le contenu serait défini avec les usagers selon leurs goûts ou leurs besoins. Une bibliothèque ambulante en quelque sorte offerte à la consultation sur place ou au prêt. Un prêt non pas en bibliothèque mais d'une bibliothèque. Il semble que ce ne soit pas le cas.

Deuxième constat, le livre numérique n'est pas perçu, dans le champ professionnel, comme étant intrinsèquement initiateur d'une évolution des pratiques de lecture mais plutôt comme s'inscrivant dans un mouvement plus large de reconfiguration des pratiques informationnelles à la lumière du numérique. Il en irait de même pour les métiers, il paraît ainsi légitime de s'interroger sur ce point.

Enfin, les personnes les plus hostiles ou réticentes au développement d'offres axées sur le numérique ne sont pas nécessairement les moins familiarisées avec l'outil. Il apparaît clairement qu'elles ont suivi des formations ou ont une expérience pratique antérieure avec d'autres outils numériques. Les causes de ces réticences sont donc probablement à chercher ailleurs.

FABRICE PIROLLI

Laboratoire CIMEOS -

Université de Bourgogne

ERIC HEILMANN

Laboratoire CIMEOS -

Université de Bourgogne 


\section{BIBLIOGRAPHIE}

Bertrand A.-M., (2004), Les bibliothèques, Paris, La découverte, collection repères, culture-communication, $118 \mathrm{p}$.

Black A., (2008), Socially controlled space or public sphere "third place"? Adult reading rooms in early British public libraries, in Koren Marian (dir.), Working for Five Star Libraries, International Perspectives on a century of public library library advocacy and development, Den Haag: Vereinigingopenbarebibliotheken, pp. 27-41.

Boullier D. et Crépel M., (2013), Pratiques de lecture et d'achat de livres numériques, étude réalisée pour le MOTif, février 2013, 114 p.

Collet L. et Pelissier M., (2012), Les bibliothèques départementales conquises par le web 2.0 ?, in Métiers de l'information, des bibliothèques et des archives, actes du $15^{\mathrm{e}}$ colloque International sur le Document Electronique, novembre 2012, Tunis, pp. 105-116.

Gauchet M., (2009), Les métiers du livre dans la crise des médiations, in Qu'est-ce qu'un livre aujourd'hui ?, pages, marges, écrans, Les cahiers de la librairie, édition La découverte, pp. 42-46.

IDATE consulting, (2013), Étude sur l'offre commerciale de livres numériques à destination des bibliothèques de lecture publique, étude réalisée pour le Ministère de la culture et de la communication, rapport final, mars 2013, $79 \mathrm{p}$.

Le Crosnier H., (2009), Pratiques de lectures à l'ère de l'ubiquité, de la communication et du partage de la connaissance, in actes du Colloque les métamorphoses numériques du livre, organisé par l'Agence régionale du Livre Paca, pp. 7-16.

MOTif, (2011), Expérimentation : prêt de liseuses en bibliothèques, un premier bilan, Bilan consultable en ligne: http://www.lemotif.fr/fr/numerique/experimentations/ pret-de-liseuses-en-bibliotheque/ \#para_un-premier-bilan, date de dernière consultation : octobre 2013.

Oldenburg R., (2001), Celebrating the Third Place, inspiring stories about the "Great Good Places" at the Heart of Our Communities, New York, Marlowe and Company, $224 \mathrm{p}$.

Institut Opinionway, (2013), Baromètre des usages du livre numérique réalisé pour les auteurs et éditeurs associés au sein de la Société Française des Intérêts des Auteurs de l'Écrit, vague 3, février 2013, 15 p.

Pernoo M., (2012), Sciences humaines et bibliothèques : quel avenir, Tracés, revue de sciences humaines, Hors-série, A quoi servent les sciences humaines (IV) ?, ENS éditions, pp. 191-203.

Pirolli F., (2010), Web 2.0 et pratiques documentaires : évolutions, tendances, perspectives, in Quoniam L. et Zimbardo P. (dirs.), Les Cahiers du Numérique, Du web 2.0 au concept 2.0 , volume $6, \mathrm{n}^{\circ} 1, \mathrm{pp} .81-96$.

Réseau CAREL, (2013), Recommandations pour le livre numérique en bibliothèque publique, http://www.reseaucarel.org/page/recommandations-pour-le-livrenumerique-en-bibliotheque-publique, date de dernière consultation : octobre 2013.

Robin C., (2011), Les livres dans l'univers numérique, Paris, La documentation française, août 2011, 159 p. 
Serres A., (2008), L'école au défi de la culture informationnelle, in Dinet J. (dir.), Usages, usagers et compétences informationnelles au XXIe siècle, Paris, Hermès Lavoisier, Chap. 2, pp. 41-70.

Wischenbart R., (2013), Global eBook, A report on market trends and developments, octobre 2013, consultable en ligne : http://www.global-ebook.com/. 
